\title{
Formación a entrenadores de fútbol base y grado de satisfacción de los deportistas
}

\section{Football coaches training and satisfaction of young athletes}

\author{
Ortín, F.J. ${ }^{*}$, Maestre, M. ${ }^{1}$ y García-de-Alcaraz, A. ${ }^{2}$ \\ 1 Universidad de Murcia \\ 2 Universidad Politécnica de Madrid
}

\begin{abstract}
Resumen: El entrenador es una de las figuras clave en la iniciación deportiva influyendo en la continuidad de la participación deportiva. En este estudio se realizó una evaluación de las verbalizaciones del entrenador en competición y un programa de asesoramiento conductual. El objetivo del trabajo fue observar los cambios en la conducta del entrenador y examinar si esto repercutía en la satisfacción de los futbolistas. Para ello, se realizó la grabación de las verbalizaciones de 3 entrenadores durante 16 partidos de competición, se aplicó una adaptación del instrumento CBAS y un autorregistro de conductas del entrenador. Los futbolistas rellenaron un cuestionario que evaluó su grado de satisfacción con respecto a la conducta de sus entrenadores. Los resultados mostraron una disminución de las conductas verbales con enfoque negativo por parte de los entrenadores, y un aumento en el grado de satisfacción de los deportistas tras la aplicación del programa de entrenamiento conductual con independencia de la categoría de competición. Estos resultados destacan la necesidad de desarrollar programas de asesoramiento en jóvenes entrenadores para favorecer la adherencia de los deportistas.
\end{abstract}

Palabras clave: psicología, deporte, fútbol, formación de entrenadores, CBAS.
Abstract: The coach is one of the main figures in sports initiation continuity of the sport participation of the players. In this study an evaluation of the verbalizations of the trainer in a competition was proposed, along with a behavioral program. The purpose was to observe the behavioral changes in the trainer and examine if these would influence the satisfaction of the footballers. The verbalizations of three trainers during sixteen competition matches where taped, an adaptation of the CBAS was applied along with a self register of the trainers behaviors and a self register of the weekly targets of 3 trainers. The players of the 3 groups filled in a form that evaluated their levels of satisfaction regarding the behavior of their trainers.

Results showed a decrease in the coaches' negative verbal behaviors, and an increase in the athletes satisfaction after the behavior training program application, no matter the level of the competition. These results highlight the need to develop training programs for coaches in order to promote the involvement of players.

Keywords: psychology, sports, football, trainer's formation, CBAS.

\section{Introducción}

Los valores sociales que desarrolla el deporte siguen siendo ampliamente destacados en la actualidad (Ruiz, Ponce, Sanz, y Valdemoros, 2015). El entorno deportivo está conformado por agentes de socialización como entrenadores, monitores, organizadores de competiciones, familias, compañeros, etc. que se convierten en un medio de influencias socializadoras y juegan un papel importante en el desarrollo y en la formación de los jóvenes que practican un deporte (Duda y Balaguer, 2007; Keegan, Spray, Harwood y Lavallee, 2010; Torregrosa et al., 2007).

Uno de los principales factores de los que depende la formación deseada de los jóvenes deportistas es el entrenador (Nuviala, León, Gálvez, y Fernández, 2007). Su comportamiento, su relación con los jugadores, así como la forma de comunicarse con ellos, son algunas de las variables que influyen en la educación de sus deportistas. La formación de los entrenadores es un factor fundamental para asegurar ciertas garantías de comportamientos adecuados frente a sus deportistas (Goldhaber, 2010; Manrique, Gea, y Álvaro, 2013).

El tipo de enseñanza usado por los entrenadores también

Dirección para correspondencia [Correspodence address]: Francisco J. Ortín.Email: ortin@um.es tiene influencia en la comunicación del entrenador y por ende, en la formación de sus jugadores (Pereira, Mesquita, Graça, y Moreno, 2010). En este sentido, la estructura de las tareas y las instrucciones de los entrenadores durante los entrenamientos, permiten a los deportistas aprender de las situaciones del juego. Por tanto, desde el punto de vista educativo, es más deseable la enseñanza-entrenamiento táctico que una enseñanza técnica (Causer y Ford, 2014; Dyson, Griffin, y Hastie, 2004), ya que provoca la formación de deportistas reflexivos y evaluadores de su práctica deportiva.

La tarea del entrenador es diseñar un ambiente tanto de entrenamientos como de competiciones, en que el aprendizaje, la mejora de la ejecución y el desarrollo del niño, y no los resultados, sea lo más importante. Tal como señala Weiss (1993) para asegurar la continuidad en el deporte, los niños necesitan ser evaluados por su mejora y su esfuerzo, y no sólo por su ejecución en comparación con la de otros deportistas. Para crear este ambiente y enseñar los fundamentos técnicos y las destrezas tácticas propias de cada deporte los entrenadores pueden utilizar dos enfoques diferentes: el enfoque positivo o el enfoque negativo.

El primero se caracteriza por recompensar las conductas deseadas de los deportistas para que éstas se produzcan con 
mayor frecuencia en el futuro. El enfoque positivo parte de la premisa de que todos los comportamientos que se valoran se aprenden. Sin embargo, el enfoque negativo intenta eliminar los errores y las conductas no deseadas de los jugadores por medio de la crítica o del castigo. Este enfoque considera que el deportista que comete menos errores es el que gana. Cuando el estilo negativo es la práctica habitual de un entrenador, las relaciones entrenador - jugadores se deterioran y aparece en los jugadores el resentimiento y la hostilidad hacia el entrenador (Sousa, Cruz, Torregrosa, Vilches, y Viladrich, 2006).

Un objetivo prioritario de los psicólogos del deporte debería ser la observación de partidos y entrenamientos, a fin de obtener datos de las interacciones que se establecen entre los niños y los entrenadores para, posteriormente, formarles y asesorarles de una manera individualizada (Sousa et al., 2006).

Los programas de formación para entrenadores se han caracterizado mayoritariamente por sesiones grupales en formato seminario. No obstante, los programas personalizados han demostrado las ventajas de adecuar los contenidos de enseñanza a las necesidades de cada entrenador (Cruz, Torregrosa, Sousa, Mora, y Viladrich, 2011) y sus efectos son más estables a largo plazo (Gilbert, Gallimore y Trudel 2009). Un ejemplo de estos programas es el Programa de Asesoramiento Personalizado a Entrenadores (PAPE; Sousa, Smith y Cruz, 2008; Sousa, Cruz, Torregrosa, Vilches y Viladrich, 2006).

El PAPE constituye una adaptación e individualización de las directrices conductuales del Coach Effectiveness Training (CET; Smith, Smoll y Curtis, 1979) por ello se intervenía de forma individual con el objetivo de incidir sobre las necesidades específicas de cada entrenador preservando su propio estilo. Inicialmente se entrevistaba a los entrenadores para conocer sus creencias y características personales y estimar en qué medida los conceptos que planteaba la intervención eran acordes con la forma de pensar de cada entrenador. Seguidamente se observaba la conducta del entrenador en los partidos mediante el Coaching Behavior Assesment System (CBAS, Smith, Smoll y Hunt, 1977), de este modo se generaba un perfil conductual en el que basar las sesiones de formación individualizadas. Los entrenadores hacían uso del establecimiento de objetivos para elegir las conductas a mejorar y planificar su consecución.

El PAPE ha demostrado su efectividad en cuanto a la mejora de las conductas que los entrenadores establecieron como objetivo prioritario. Específicamente un aumento en aquellos comportamientos que suponían la implementación de conductas de apoyo, como el refuerzo o el ánimo, y una disminución de las conductas punitivas. Estudios de revisión recientes (Cruz, et al., 2011; Langan, Blake, y Lonsdale, 2013) han apoyado la línea de intervención del PAPE, destacando la importancia de las intervenciones individualizadas.
Considerando los beneficios asociados a los programas individualizados con entrenadores (Cruz et al., 2011; Langan et al., 2013; Su y Reeve, 2011) en el presente estudio se propone el diseño de un programa de formación a entrenadores a través de una evaluación de las verbalizaciones del entrenador en un contexto de competición.

El objetivo del trabajo es observar los cambios en la conducta del entrenador y examinar si ello repercute en la satisfacción de los deportistas jóvenes en relación a dicha conducta; la hipótesis planteada se basa en que tras el programa de formación y asesoramiento conductual e individual impartido a los entrenadores, éstos aumentarán la frecuencia de las conductas que corresponden al enfoque positivo, disminuirán la frecuencia de las conductas que corresponden al enfoque negativo y, el grado de satisfacción de sus jugadores aumentará; ya que los estudios revisados por Chelladurai (1993) confirman que los deportistas se muestran más satisfechos cuando el tipo de liderazgo del entrenador consiste en valorar la habilidad y los esfuerzos de los miembros del equipo con feedback positivo que recompense la ejecución correcta.

\section{Método}

\section{Participantes}

El presente estudio se realizó con 3 entrenadores de fútbol pertenecientes a una escuela de fútbol de la Región de Murcia, que participaban en un campeonato de liga de fútbol regional en la categoría de infantil, cadete y juvenil. Los entrenadores y deportistas que participaron en este estudio conforman una muestra incidental, no se realizó una aleatorización para la selección de la misma.

El entrenador 1 tiene 24 años de edad, 7 años de experiencia como entrenador de fútbol y 1 año como coordinador de fútbol, correspondió al entrenador del equipo de la categoría infantil. El entrenador 2 tiene 27 años de edad y 5 años de experiencia como entrenador de fútbol, correspondió al entrenador del equipo de la categoría cadete. El entrenador 3 tiene 38 ańos de edad, 15 ańos de experiencia como entrenador de fútbol y 3 temporadas como director deportivo, correspondió al entrenador del equipo de la categoría juvenil. La media de edades de los tres entrenadores al inicio del estudio era 29 años (DT $=6,1)$ y una media de 9 temporadas (DT $=4,32)$ de experiencia como entrenadores de fútbol.

Asimismo, también formaron parte de este estudio los deportistas que conformaban cada uno de los equipos mencionados: infantil, cadete y juvenil. Un total de 36 futbolistas distribuidos en: 9 jugadores de categoría infantil, 15 jugadores de categoría cadete y 12 jugadores de categoría juvenil. 


\section{Variables}

Se procedió a la medida de tres variables: las conductas verbales de los entrenadores, autorregistros conductuales de los entrenadores y grado de satisfacción de los futbolistas.

Las verbalizaciones de los entrenadores hacen referencia a cada una de las palabras que pronuncia cada uno de los entrenadores durante los partidos de competición. Para su medida se utiliza el instrumento CBAS y se realiza una grabación de voz de cada uno de ellos durante los 90 minutos que dura cada partido. Cada palabra/frase/comentario se codifica en la categoría correspondiente y al término del partido se obtiene una puntuación final sobre la frecuencia con la que cada entrenador ha realizado cada conducta verbal.

Los autorregistros conductuales reflejan la reflexión que cada entrenador desarrolla acerca de sus propias conductas interactivas con los jugadores durante los entrenamientos. Cada entrenador dispone del autorregistro en cada entrenamiento y al finalizar éste tienen que puntuar sus propias conductas en una escala tipo Likert de 1 a 5 en función de su realización y frecuencia.

El grado de satisfacción de los deportistas se refiere al grado de satisfacción que presenta cada deportista con su entrenador en relación a su actitud y su comportamiento en el contexto deportivo.

\section{Instrumentos}

Para la realización de este estudio se han empleado tres grabadoras de voz de marca Panasonic junto con un micrófono de solapa, una para cada entrenador, utilizadas para grabar las verbalizaciones de los entrenadores en cada uno de los partidos de competición que pasaron a formar parte de la investigación.

El Sistema de Evaluación de la Conducta del Entrenador (CBAS, Smith, et al., 1977a). Este instrumento registra dos tipos de conductas: conductas reactivas y conductas espontáneas. Se ha utilizado una adaptación de este instrumento ya que los datos con los que trabajamos no fueron visuales sino que fueron únicamente verbales impidiendo la aplicación de algunas categorías. Por ello, de las 13 categorías que presenta el instrumento original en este estudio se trabajó con 9: Refuerzo, Ánimo al Error, Instrucción Técnica al Error, Punición/Castigo, Instrucción Técnica Punitiva, Instrucción Técnica General, Ánimo General, Organización y Comunicación General. Se utilizó para registrar los comportamientos de los entrenadores en un contexto de competición.

Se ha empleado un autorregistro a rellenar por los entrenadores sobre conductas del entrenador en los entrenamientos (adaptación Buceta, 1998). Se mide en una escala tipo Likert de 1 a 5 puntos. Se aplicó con el objetivo de que los entrena- dores se autoevaluaran sobre el uso de determinadas conductas en el entrenamiento.

Por último, se desarrolló un cuestionario de satisfacción ad hoc destinado a los jugadores de los tres equipos. Se aplicó para conocer el grado de satisfacción que presentaban los jugadores de los tres equipos con respecto a la conducta de su entrenador.

\section{Procedimiento}

El presente estudio se desarrolló en 3 fases. La primera fase consistió en elaborar una línea base tanto de las conductas que realizaban los entrenadores como del grado de satisfacción que presentaban los jugadores con respecto a la conducta de su entrenador. Dentro de esta primera fase, se realizó la primera grabación de las verbalizaciones de los entrenadores durante partidos de competición. Concretamente, se produjo la grabación de 3 partidos en categoría infantil, 3 partidos en categoría cadete y 2 partidos en categoría juvenil. Para ello, se le entregó a cada entrenador una grabadora junto con un micrófono de solapa y se les recordó antes de cada partido que iniciaran la grabación. Una vez que obtuvimos todos los datos, se procedió a categorizarlos en función de las nueve categorías del CBAS que se decidieron mantener: Refuerzo, Ánimo al Error, Instrucción Técnica al Error, Punición/Castigo, Instrucción Técnica Punitiva, Instrucción Técnica General, Ánimo General, Organización y Comunicación General.

La segunda fase consistió en una reunión individualizada con cada uno de los tres entrenadores. En esta reunión se les presentaron los datos que conformaban la línea base de sus comportamientos durante los partidos de competición, extraídos de la grabación de las verbalizaciones. Es decir, se les aportó retroalimentación y se les proporcionó asesoramiento individual acerca de aquellas conductas que consideramos que podrían mejorar. El objetivo fue que los entrenadores tomaran conciencia de sus comportamientos y analizaran, junto con el investigador, las conductas que debían mantener y potenciar y cuáles debían evitar en su perfil conductual. Por otro lado, los entrenadores recibieron asesoramiento continuo sobre aspectos conductuales relacionados con el autorregistro diario.

La tercera y última fase consistió en volver a evaluar el grado de satisfacción de los deportistas y, de nuevo, las verbalizaciones de los entrenadores en un contexto de competición Esta nueva evaluación se llevó a cabo dos meses después del feedback y asesoramiento conductual indicado en la fase anterior. Para ello, se volvió a utilizar el cuestionario elaborado ad hoc que mide el grado de satisfacción de los jugadores con respecto a la conducta del entrenador, y se volvió a grabar las verbalizaciones de los entrenadores durante los partidos de competición. Concretamente, se produjo la grabación de 3 partidos en categoría infantil, 3 partidos en categoría cadete 
y 2 partidos en categoría juvenil. Una vez que obtuvimos todos los datos, se procedió a categorizarlos en función de las nueve categorías del CBAS que se comentaron anteriormente. El objetivo de esta fase fue la comprobación de la posible influencia del asesoramiento, es decir, si los entrenadores habían mejorado en cuanto a sus conductas y si la satisfacción de los jugadores había aumentado.

Por último, los entrenadores obtuvieron de nuevo feedback sobre los datos de las grabaciones, incluyendo la variación de las diferentes verbalizaciones, el aumento o no del grado de satisfacción de los deportistas y la percepción de estos sobre el cambio conductual.

\section{Análisis estadístico}

Se realizó un análisis descriptivo de las conductas verbales de los entrenadores, y de la reflexión de entrenadores (auto registros conductuales) y jugadores (grado de satisfacción) antes y después de la aplicación del programa de formación y asesoramiento conductual. Se calculó la media y la desviación típica para cada variable analizada. Sólo se realizó un análisis inferencial para el estudio de la reflexión de los entrenadores antes y después de la aplicación del programa. Se utilizó el Test de Wilcoxon para muestras relacionadas, estableciendo un nivel de significación de $\mathrm{p}<.05$.

\section{Resultados}

Los datos acerca de las verbalizaciones de los entrenadores antes y después de la aplicación del programa de formación y asesoramiento conductual (Tabla 1), mostraron en la categoría infantil un aumento en la información relacionada con el refuerzo, el ánimo al error, el ánimo general y la organización; un descenso en la información relacionada con el castigo (punición e instrucción técnica punitiva), la instrucción técnica al error y la comunicación general; y un mantenimiento de la instrucción técnica general. En la categoría cadete solo se observó un aumento en la información relacionada con el refuerzo. El resto de variables presentaron una disminución. En la categoría juvenil se encontraron ligeros incrementos en la información relacionada con el ánimo al error, ánimo general y organización; un descenso en la información relacionada con el refuerzo, punición/castigo, instrucción técnica punitiva e instrucción técnica general; y un mantenimiento de la instrucción técnica al error y la comunicación general (inexistente en este último caso).

Con independencia de la categoría, se mostró un descenso en la información relacionada con el castigo (punición e instrucción técnica punitiva) tras la aplicación del programa de formación y asesoramiento conductual.

Tabla 1. Verbalización de los entrenadores antes y después del programa de formación y asesoramiento conductual (PFAC)

\begin{tabular}{|c|c|c|c|c|c|c|c|c|c|c|c|c|}
\hline & \multirow{2}{*}{\multicolumn{4}{|c|}{ Infantil (sub-14. $70 \mathrm{~min}$ ) }} & \multirow{2}{*}{\multicolumn{4}{|c|}{ Cadete (sub-16. $80 \mathrm{~min})$}} & \multirow{2}{*}{\multicolumn{4}{|c|}{ Juvenil (sub-19.90 min) }} \\
\hline & & & & & & & & & & & & \\
\hline & \multicolumn{2}{|c|}{$\begin{array}{l}\text { Pre-PFAC } \\
\text { (3 partidos) }\end{array}$} & \multicolumn{2}{|c|}{$\begin{array}{l}\text { Post-PFAC } \\
\text { (3 partidos) }\end{array}$} & \multicolumn{2}{|c|}{$\begin{array}{l}\text { Pre-PFAC } \\
\text { (3 partidos) }\end{array}$} & \multicolumn{2}{|c|}{$\begin{array}{l}\text { Post-PFAC } \\
\text { (3 partidos) } \\
\end{array}$} & \multicolumn{2}{|c|}{$\begin{array}{l}\text { Pre-PFAC } \\
\text { (2 partidos) }\end{array}$} & \multicolumn{2}{|c|}{$\begin{array}{l}\text { Post-PFAC } \\
\text { (2 partidos) }\end{array}$} \\
\hline & $\mathrm{M}$ & DT & $\mathrm{M}$ & DT & M & DT & $\mathrm{M}$ & DT & M & $\mathrm{DT}$ & $\mathrm{M}$ & DT \\
\hline Refuerzo & 48,33 & 13,43 & 50,33 & 5,86 & 64,00 & 23,07 & 90,33 & 9,07 & 80,00 & 16,97 & 69,50 & 23,33 \\
\hline Ánimo al error & 13,33 & 5,86 & 26,00 & 6,56 & 15,00 & 3,46 & 9,33 & 2,52 & 12,50 & 0,71 & 14,50 & 10,61 \\
\hline Instrucción técnica al error & 52,00 & 10,54 & 31,33 & 3,51 & 26,33 & 5,03 & 22,33 & 2,52 & 26,50 & 2,12 & 26,50 & 4,95 \\
\hline Punición/Castigo & 16,33 & 4,04 & 8,33 & 1,15 & 19,67 & 5,13 & 7,67 & 1,53 & 13,50 & 2,12 & 8,50 & 2,12 \\
\hline Instrucción técnica punitiva & 7,00 & 2,00 & 3,33 & 2,08 & 2,67 & 1,53 & 0,00 & 0,00 & 5,50 & 0,71 & 1,00 & 1,41 \\
\hline Instrucción técnica general & 66,33 & 21,20 & 66,33 & 7,09 & 105,00 & 10,44 & 90,00 & 3,61 & 101,50 & 6,36 & 68,50 & 3,54 \\
\hline Ánimo general & 18,33 & 6,66 & 35,67 & 6,03 & 57,00 & 10,82 & 56,00 & 27,18 & 28,50 & 17,68 & 29,00 & 1,41 \\
\hline Organización & 79,33 & 14,64 & 86,33 & 9,61 & 115,67 & 39,93 & 98,67 & 38,18 & 92,00 & 26,87 & 99,00 & 19,80 \\
\hline Comunicación general & 0,67 & 1,15 & 0,33 & 0,58 & 6,67 & 6,66 & 1,00 & 1,00 & 0,00 & 0,00 & 0,00 & 0,00 \\
\hline
\end{tabular}

Nota PFAC $=$ Programa de Formación y Asesoramiento Conductual. $\mathrm{M}=$ media aritmética. $\mathrm{DT}=$ Desviación típica.

En la Tabla 2 se muestran los autorregistros realizados por los entrenadores antes y después de la aplicación del programa de formación y asesoramiento conductual. El entrenador de categoría infantil presentó un aumento estadísticamente significativo de la información relacionada con la explicación del objetivo del ejercicio, las reglas del ejercicio y la claridad de los objetivos establecidos. También se encontró un aumento estadísticamente significativo en el grado de consecución de los objetivos de aprendizaje por parte de los deportistas, en el empleo de un plan para alcanzar los objetivos y en el seguimiento exhaustivo del deportista, así como en la utilización de material para los ejercicios y de sistemas de premios. Por el contrario, hubo un descenso estadísticamente significativo en la evaluación de la consecución de objetivos. 
El entrenador de categoría cadete mostró un aumento estadísticamente significativo en las variables que indican que el deportista sabe lo que el entrenador espera de éste y qué debe hacer el deportista para mejorar, así como en la evaluación de la consecución de objetivos. También se observó un descenso estadísticamente significativo en relación a la corrección y ánimos tras el error.

El entrenador de categoría juvenil registró un aumento es- tadísticamente significativo en las variables que indican que el deportista sabe lo que el entrenador espera de éste, lo que tiene que mejorar y qué debe hacer el deportista para mejorar, así como el grado de consecución de los objetivos de aprendizaje por parte de los deportistas. También se observó un aumento estadísticamente significativo en relación a la corrección y ánimos tras el error.

Tabla 2. Auto registros conductuales del entrenador antes y después del programa de formación y asesoramiento conductual (PFAC).

\begin{tabular}{|c|c|c|c|c|c|c|}
\hline & \multirow{2}{*}{\multicolumn{2}{|c|}{ Infantil (sub-14) }} & \multirow{2}{*}{\multicolumn{2}{|c|}{ Cadete (sub-16) }} & \multirow{2}{*}{\multicolumn{2}{|c|}{ Juvenil (sub-19) }} \\
\hline & & & & & & \\
\hline & $\begin{array}{l}\text { Pre-PFAC } \\
\text { (10 entren.) }\end{array}$ & $\begin{array}{c}\text { Post-PFAC } \\
(8 \text { entren.) }\end{array}$ & $\begin{array}{c}\text { Pre-PFAC } \\
\text { (10 entren.) }\end{array}$ & $\begin{array}{l}\text { Post-PFAC } \\
(8 \text { entren.) }\end{array}$ & $\begin{array}{c}\text { Pre-PFAC } \\
\text { (10 entren.) }\end{array}$ & $\begin{array}{c}\text { Post-PFAC } \\
(8 \text { entren.) }\end{array}$ \\
\hline & $\mathrm{M} \pm \mathrm{DT}$ & $\mathrm{M} \pm \mathrm{DT}$ & $\mathrm{M} \pm \mathrm{DT}$ & $\mathrm{M} \pm \mathrm{DT}$ & $\mathrm{M} \pm \mathrm{DT}$ & $\mathrm{M} \pm \mathrm{DT}$ \\
\hline Explico objetivo del ejercicio & $3,9 \pm 0,99$ & $5 \pm 0^{*}$ & $4,8 \pm 0,42$ & $4,87 \pm 0,35$ & $4,9 \pm 0,32$ & $5 \pm 0$ \\
\hline Explico reglas del ejercicio & $3,9 \pm 0,57$ & $4,87 \pm 0,35^{*}$ & $5 \pm 0$ & $4,62 \pm 0,52$ & $5 \pm 0$ & $5 \pm 0$ \\
\hline Miro al deportista cuando me dirijo a él & $5 \pm 0$ & $5 \pm 0$ & $5 \pm 0$ & $4,75 \pm 0,46$ & $5 \pm 0$ & $5 \pm 0$ \\
\hline Utilizo preguntas para que den respuesta & $3,7 \pm 0,95$ & $4,37 \pm 0,52$ & $4,3 \pm 0,67$ & $4,5 \pm 0,53$ & $4,8 \pm 0,42$ & $5 \pm 0$ \\
\hline Animo al deportista & $5 \pm 0$ & $4,75 \pm 0,46$ & $4,8 \pm 0,42$ & $4,5 \pm 0,53$ & $4,6 \pm 0,52$ & $5 \pm 0$ \\
\hline Indico lo que se hace bien & $4,6 \pm 0,52$ & $4,62 \pm 0,52$ & $5 \pm 0$ & $4,75 \pm 0,46$ & $4,6 \pm 0,52$ & $5 \pm 0$ \\
\hline Corrijo y animo después & $4,8 \pm 0,42$ & $4,37 \pm 0,52$ & $5 \pm 0$ & $4,5 \pm 0,53^{*}$ & $4,5 \pm 0,53$ & $5 \pm 0^{*}$ \\
\hline Doy oportunidad a que me pregunten & $4,5 \pm 0,71$ & $4,5 \pm 0,53$ & $4,9 \pm 0,32$ & $4,75 \pm 0,46$ & $4,9 \pm 0,32$ & $5 \pm 0$ \\
\hline El deportista sabe lo que espero de él/ella & $4,1 \pm 0,87$ & $4,37 \pm 0,52$ & $4,2 \pm 0,63$ & $4,75 \pm 0,46^{*}$ & $4 \pm 0$ & $4,75 \pm 0,46^{*}$ \\
\hline El deportista sabe lo que tiene que mejorar & $3,7 \pm 0,82$ & $4,25 \pm 0,46$ & $4,3 \pm 0,67$ & $4,75 \pm 0,46$ & $4 \pm 0$ & $4,75 \pm 0,46^{*}$ \\
\hline El deportista sabe qué tiene que hacer para mejorar & $3,6 \pm 0,52$ & $4,25 \pm 0,46$ & $4 \pm 0,67$ & $4,75 \pm 0,46^{*}$ & $4 \pm 0$ & $4,75 \pm 0,46^{*}$ \\
\hline Los deportistas consiguen los objetivos de aprendizaje & $3,5 \pm 0,53$ & $4,25 \pm 0,46^{*}$ & $4,3 \pm 0,67$ & $4,12 \pm 0,35$ & $4,1 \pm 0,57$ & $4,75 \pm 0,46^{*}$ \\
\hline Tengo objetivos claros & $2,7 \pm 0,48$ & $4,25 \pm 0,46^{* *}$ & $4,7 \pm 0,67$ & $4,5 \pm 0,53$ & $4,7 \pm 0,48$ & $5 \pm 0$ \\
\hline Tengo un plan para que consigan objetivos & $2,7 \pm 0,48$ & $4,25 \pm 0,46^{* *}$ & $4,5 \pm 0,85$ & $4,5 \pm 0,53$ & $4,7 \pm 0,48$ & $5 \pm 0$ \\
\hline Evalúo consecución de objetivos & $5 \pm 0$ & $4,37 \pm 0,52^{* *}$ & $3,9 \pm 0,74$ & $4,75 \pm 0,46^{*}$ & $4,7 \pm 0,48$ & $5 \pm 0$ \\
\hline Utilizo el material disponible para el ejercicio & $4,5 \pm 0,53$ & $5 \pm 0^{*}$ & $3,8 \pm 0,63$ & $3,87 \pm 0,83$ & $5 \pm 0$ & $5 \pm 0$ \\
\hline Utilizo sistema de premios (refuerzos) & $2,7 \pm 0,48$ & $5 \pm 0^{* *}$ & $4,3 \pm 0,82$ & $4,5 \pm 0,53$ & $4,7 \pm 0,48$ & $5 \pm 0$ \\
\hline Llevo un seguimiento de cada deportista (fichas, etc.) & $4,4 \pm 0,52$ & $5 \pm 0^{*}$ & $5 \pm 0$ & $5 \pm 0$ & $4,9 \pm 0,32$ & $5 \pm 0$ \\
\hline
\end{tabular}

Nota PFAC $=$ Programa de Formación y Asesoramiento Conductual. Entren: entrenamientos. $\mathrm{M} \pm \mathrm{DT}=$ media aritmética y desviación típica. ${ }^{*}$ Diferencia significativa inferior a $.05 .{ }^{* *}$ Diferencia significativa inferior a .01.

En la Tabla 3 se indica el grado de satisfacción de los jugadores antes y después de la aplicación del programa de formación y asesoramiento conductual. Se observó un incremento del grado de satisfacción general (me siento muy satisfecho), y del grado de satisfacción en relación a las tareas de entrena- miento (ejercicios útiles y divertidos), a la actitud del entrenador (agradable y bueno), y a la utilidad del deporte (relación con amigos, conseguir metas y ser feliz) en todas las categorías de competición. 
Tabla 3. Grado de satisfacción de los jugadores antes y después del programa de formación y asesoramiento conductual (PFAC).

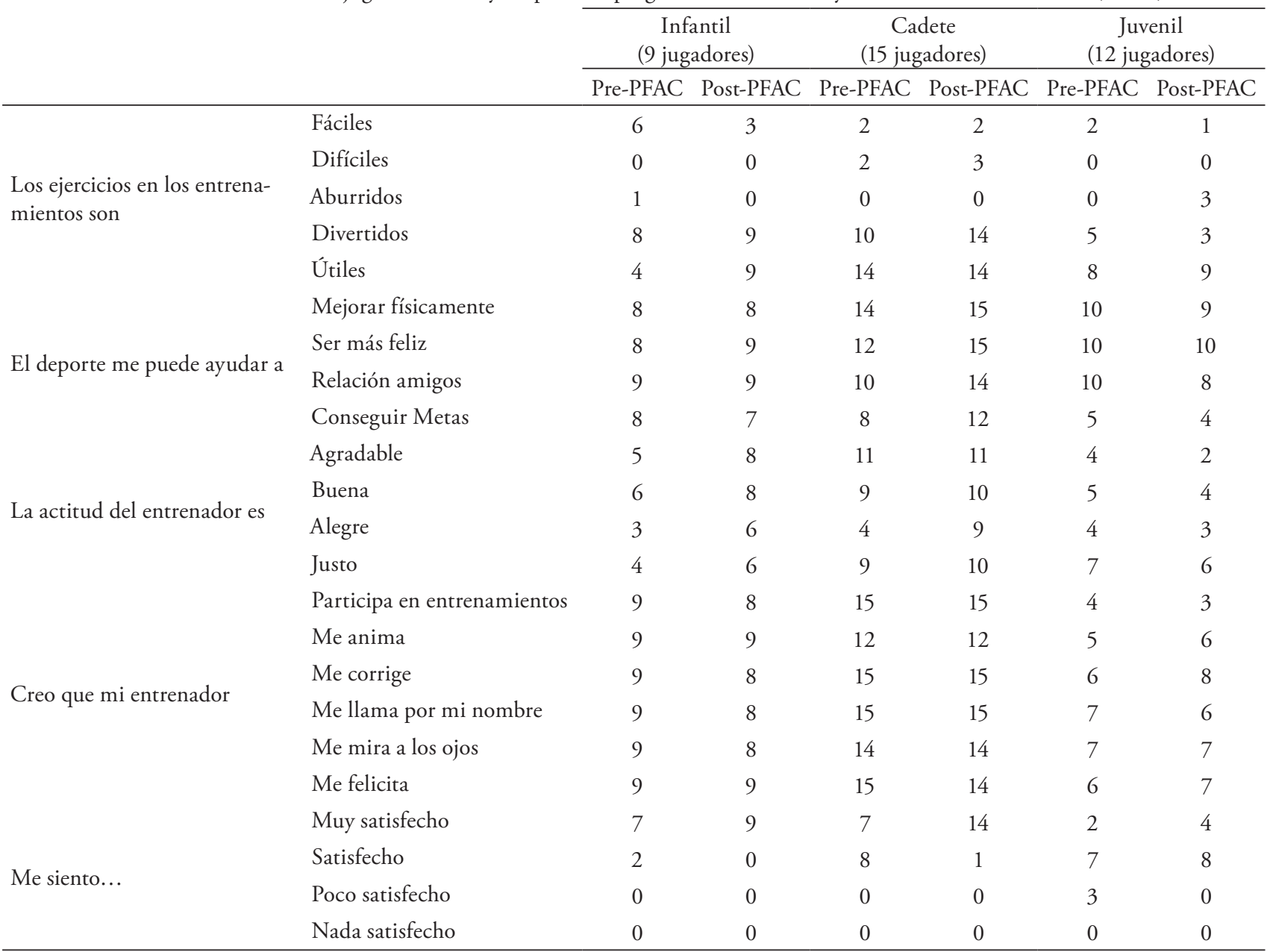

Nota. PFAC = Programa de Formación y Asesoramiento Conductual.

\section{Discusión y Conclusiones}

Tras el análisis de los resultados, se puede concluir que realizar un seguimiento de la conducta del entrenador y aportarle recomendaciones específicas en sesiones individualizadas puede ayudar a los entrenadores a presentar conductas positivas con sus jugadores. Similares resultados que los estudios de revisión recientes (Cruz, et al., 2011; Langan, Blake, y Lonsdale, 2013) donde se ha destacado la importancia de las intervenciones individualizadas.

En línea con otros trabajos (Cruz et al., 2011; Sousa et al., 2008; Sousa et al., 2006) y en concordancia con nuestra hipótesis planteada, los tres entrenadores disminuyeron de manera estadísticamente significativa la frecuencia de las conductas verbales punitivas y se aumentó la frecuencia de las conductas del estilo positivo después de recibir el programa de formación y asesoramiento conductual. Tal como espe- cifican González, Márquez y Tabernero (2000) los deportistas de fútbol base consideran al entrenador como aspecto esencial de su participación deportiva. Es por eso que en la conducta del entrenador en el deporte de iniciación debe predominar un estilo positivo, tanto en entrenamientos como en partidos de competición, para motivar a sus jugadores y poder contribuir a que sean adultos activos.

Asimismo, parece que puede existir una posible relación entre un aumento de la frecuencia de las conductas del enfoque positivo y una disminución de la frecuencia de las conductas que corresponden al enfoque negativo por parte de los entrenadores con un aumento en el grado de satisfacción de sus deportistas. Estas conclusiones seguirían la línea de los estudios revisados por Chelladurai (1993) que confirman que los deportistas se muestran más satisfechos cuando el tipo de liderazgo del entrenador consiste en valorar la habilidad y los esfuerzos de los miembros del equipo con feedback positivo 
que recompense la ejecución correcta.

Los tres entrenadores evaluaron como muy útil y relevante la reflexión acerca de sus propias conductas interactivas con los jugadores durante los entrenamientos para su labor profesional. Ya que comenzaron a realizar conductas positivas con sus jugadores que habían dejado de efectuar con los años de experiencia como entrenadores (Cruz, Sousa, Torregrosa, Viladrich y Vilches, 2006).

En el deporte en estas edades la actuación no solo del entrenador sino también la de los padres juega un papel muy importante conformando el llamado triángulo deportivo. Así, como señala Cruz (2001), en el deporte base el psicólogo debe priorizar el asesoramiento fundamentalmente a entrenadores y padres. Esto dará lugar a que los deportistas asocien el deporte con una experiencia positiva y, por lo tanto, a que disminuya la tasa de abandono del deporte en edades tempranas, como se comprueba en el trabajo de Barnett et al. (1992).

Los programas personalizados han demostrado las ventajas de adecuar los contenidos de enseñanza a las necesidades de cada entrenador (Cruz, Torregrosa, Sousa, Mora, y Viladrich, 2011) y sus efectos son más estables a largo plazo (Gilbert, Gallimore y Trudel 2009), sin embargo deben seguir realizándose estudios en esta línea con programas de formación y asesoramiento conductual no solo a entrenadores sino también dirigidos a las familias, así como a organizadores de competiciones deportivas para niños, árbitros y demás figuras significativas para el deporte de iniciación.

\section{Referencias}

1. Barnett, N.P., Smoll, F.L. y Smith, R.E. (1992). Effects of enhancing coach-athlete relationships on youth sport attrition. The Sport Psychologist, 6, 111-127.

2. Buceta, J.M. (1998). Psicología del entrenamiento deportivo. Madrid: Dikynson.

3. Causer, J., y Ford, P. R. (2014). «Decisions, decisions, decisions»: transfer and specificity of decision-making skill between sports. Cognitive process, $15,385-389$.

4. Chelladurai, P. (1993). Leadership. En R.N. Singer, M. Murphy y L. K. Tennant (Eds.) Handbook of sport psychology (pp. 647-671). Nueva York: McMillan.

5. Cruz, J. (2001). Factores motivacionales en el deporte infantil y asesoramiento psicológico a entrenadores y padres. En J. Cruz (Ed.), Psicología del Deporte (pp. 147-176). Primera Reimpresión. Madrid: Síntesis.

6. Cruz, J.; Torregrosa, M., Sousa, C., Mora, A. y Viladrich, C. (2011). Efectos conductuales de programas personalizados de asesoramiento a entrenadores en estilo de comunicación y clima motivacional. Revista de Psicología del Deporte. 20/1, 179-195.

7. Duda, J. L., y Balaguer, I. (2007). Coach-created motivacional climate. En S. Jowet y D. Lavallee (Eds.). Social Psychology in Sport (pp117-130). Champaign, IL: Human Kinetics.

8. Dyson, B., Griffin, L. L., y Hastie, P. (2004). Sport Education, tactical games, and cooperative learning: Theroetical and pedagogical considerations. Quest, 56, 226-240.

9. Gilbert, W. D., Gallimore, R. y Trudel, P. (2009). A learning community approach to coach development in youth sport. Journal of Coaching Education, 2(2), 1-21.

10. Goldhaber, D. (2010). Licensure Tests: Their Use and Value for Increasing Teacher Quality. En Kennedy, M. (Ed.), Teacher Assessment and the Quest for Teacher Quality: A Handbook (pp. 133-147). San Francisco: Jossey-Bass.

11. González, G., Tabernero, B., y Márquez, S. (2000). Análisis de los motivos para participar en fútbol y en tenis en la iniciación deportiva. $M o$ tricidad, 6, 47-66.

12. Keegan, R., Spray, C., Harwood, C. y Lavallee, D. (2010). The motivational atmosphere in youth sport;coach, parent, and peer influences on motivation in specializing sport participants. Journal of Applied Sport Psychology, 22 (1), 87-105.

13. Langan, E., Blake, C. y Lonsdale, C. (2013). Systematic review of interpersonal coach education interventions on athlete outcomes. Psychology of Sport and Exercise, 14, 37-49.

14. Manrique, J. C., Gea, J. M., y Álvaro, M. (2013). Perfil y expectativas del técnico de deporte escolar en el municipio de Segovia (España). Revista Internacional de Medicina y Ciencias de la Actividad Física y el Deporte, 13(50), 367-387. Recuperado de http://cdeporte.rediris.es/revista/ revista50/artperfil383.pdf

15. Nuviala, A., León, J.A., Gálvez, J., y Fernández, A. (2007) Qué actividades deportivas escolares queremos. Qué técnicos tenemos. Revista Internacional de Medicina y Ciencias de la Actividad Física y el Deporte, $7(25), 1-9$.

16. Pereira, F, Mesquita, I., Graça, A., y Moreno, M. P. (2010). Análisis multidimensional del feedback pedagógico en entrenamiento en voleibol. Revista Internacional de Medicina y Ciencias de la Actividad Física y el Deporte 10(38) 181-202. Recuperado de http://cdeporte.rediris.es/ revista/revista38/artanalisis146.htm

17. Ruiz, J. V., Ponce, A., Sanz, E., y Valdemoros, M. A. (2015). La educación en valores desde el deporte: investigación sobre la aplicación de un programa integral en deportes de equipo. Retos, 28, 270-276.

18. Smith, R. E., Smoll, F. L. y Hunt, E. B. (1977a). A system for the behavioral assessment of athletic coaches. Research Quarterly, 48, 401-407.

19. Smith, R.E.; Smoll, F. L. y Curtis, B. (1979). Coach effectiveness training: a cognitive-behavioral approach to enhancing relationship skills in youth aports coaches. Journal of Sport Psychology, 1, 59-75. Traducción en J. Cruz y J. Riera (Eds.), Psicología del deporte: aplicaciones y perspectivas (pp. 118-139). Barcelona: Martínez Roca.

20. Sousa, C., Cruz, J., Torregrosa, M., Vilches, D., y Viladrich, C. (2006). Evaluación conductual y programa de asesoramiento personalizado a entrenadores (PAPE) de deportistas. Revista de Psicología del Deporte, 15, 263-278.

21. Sousa, C., Smith, R. E. y Cruz, J. (2008). An individualized behavioral goal-setting program for coaches. Journal of Clinical Sport Psychology, 2, 258-277.

22. Su, Y-L. y Reeve, J. (2011). A meta-analysis of the effectiveness of intervention programs designed to support autonomy. Educational Psychology Review, 23, 159-188. doi:10.1007/s10648-010-9142-7.

23. Torregrosa, M., Cruz, J., Sousa, C., Viladrich, C., Villamarín, F., García Mas, A. y Palou, P. (2007). La influencia de padres y madres en el compromiso deportivo de futbolistas jóvenes. Revista Latinoamericana de Psicología, 39, 227-237.

24. Weiss, M. R. (1993). Psychological effects of intensive sport participation on children and youth: self-esteem and motivation. En B. Cahill y A Pearl (eds.). Perspectives on intensive participation in youth sports (pp. 39-69). Champaign, IL.: Human Kinetics. 